PROCEEDINGS OF THE AMERICAN MATHEMATICAL SOCIETY

Volume 124, Number 8, August 1996

\title{
CRUMPLED LAMINATIONS AND MANIFOLDS OF NONFINITE TYPE
}

\author{
R. J. DAVERMAN AND F. C. TINSLEY \\ (Communicated by James E. West)
}

\begin{abstract}
Using a group-theoretic construction due to Bestvina and Brady, we build $(n+1)$-manifolds $W$ which admit partitions into closed, connected $n$-manifolds but which do not have finite homotopy type.
\end{abstract}

At the heart of this note is an example due to Bestvina and Brady [1] of an almost finitely presented group which is not finitely presented. Specifically, they describe a finitely presented group $G$ with a perfect normal subgroup $P$ such that $G / P$ is not finitely presented (i.e., $P$ fails to be the normal closure in $G$ of a finite set); furthermore, $P$ itself is expressed as an infinite free product $*_{i} P_{i}$ of finitely presented groups $P_{i}$, which happen to be pairwise isomorphic.

For each positive integer $m$ let $\Gamma_{m}$ denote $P_{1} * P_{2} * \cdots * P_{m} \subset P$, and let $N_{m}$ denote the normal closure of $\Gamma_{m}$ in $G$. Then

$$
N_{1} \subset N_{2} \subset \cdots \subset N_{m} \subset N_{m+1} \subset \cdots
$$

and $P=\bigcup N_{m}$. Set $G_{m}^{\prime}=G / N_{m}$. Note the existence of natural projections $\psi_{m}: G_{m}^{\prime} \rightarrow G_{m+1}^{\prime}$; the direct limit of $\left\{\psi_{m}\right\}$ is $G / P$. Since $P=\bigcup N_{m}$ fails to be finitely generated as a normal subgroup, infinitely many of $\left\{\psi_{m}\right\}$ must have nontrivial kernel. This answers Questions 3.4 and 3.5 of [2]. We use it here to describe crumpled laminations $p: W \rightarrow \mathbb{R}$ on manifolds $W$ which do not have finite homotopy type, answering Question 3.1 of [2] negatively, and illustrating the sharpness of the main result (Theorem 1.1) there.

Recall that a crumpled lamination on an $(n+1)$-manifold $W$ is a closed map $p$ of $W$ to an interval $J$ (possibly noncompact) such that each $p^{-1}(t)(t \in J)$ is a closed, connected $n$-manifold.

Given a compact $n$-manifold $M, n \geq 5$, and a finitely generated, perfect subgroup $H$ of $\pi_{1}(M)$, the mapping cylinder construction of [3] provides a map $f: M \rightarrow M^{\prime}$ from $M$ onto another $n$-manifold $M^{\prime}$ and a compact $(n+1)$-dimensional cobordism $\left(W, M, M^{\prime}\right)$, where the $(n+1)$-manifold $W$ is obtained from the mapping cylinder of $f$ by attaching a collar $M^{\prime} \times[1,2]$ to the obvious copy of $M^{\prime}$; in addition, here the inclusion $M^{\prime} \rightarrow W$ is a homotopy equivalence, $\pi_{1}\left(M^{\prime}\right)$ is isomorphic to the quotient of $G$ by $N(H)$, the normal closure of $H$, and inclusion $M \rightarrow W$ induces the obvious projection $G \rightarrow G / N(H)$. Since $W$ is determined as the disjoint union

Received by the editors November 19, 1995 .

1991 Mathematics Subject Classification. Primary 57N15, 57N70; Secondary 55P15, 54B15.

Key words and phrases. Crumpled lamination, cobordism, mapping cylinder, acyclic map, finitely presented group, perfect subgroup, almost finitely presented group.

(C)1996 American Mathematical Society 
of $M \times[0,1)$ and $M^{\prime} \times[1,2]$, it possesses a crumpled lamination derived from an obvious map $p: W \rightarrow[0,2]$ having $n$-manifolds as point preimages.

For $n \geq 5$ name a closed $n$-manifold $M_{0}$ for which $\pi_{1}\left(M_{0}\right) \cong G$. The mapping cylinder construction provides a laminated cobordism $\left(W_{1}, M_{0}, M_{1}\right)$ such that $M_{1} \rightarrow W_{1}$ is a homotopy equivalence, $\pi_{1}\left(M_{1}\right) \cong \pi_{1}\left(W_{1}\right) \cong G_{1}^{\prime}$, and the inclusion $M_{0} \rightarrow W_{1}$ induces the natural projection $\psi_{0}: G \rightarrow G_{1}^{\prime}=G / N_{1}$. Applying the construction recursively, we obtain successive laminated cobordisms $\left(W_{m}, M_{m-1}, M_{m}\right)$ such that $M_{m} \rightarrow W_{m}$ is a homotopy equivalence, $\pi_{1}\left(M_{m}\right) \cong \pi_{1}\left(W_{m}\right) \cong G_{m}^{\prime}$, and the inclusion $M_{m-1} \rightarrow W_{m}$ induces $\psi_{m-1}: G_{m-1}^{\prime} \rightarrow G_{m}^{\prime}$. We regard distinct $W_{i}, W_{j}$ as intersecting only if $i=j \pm 1$ and then $W_{i} \cap W_{i+1}=M_{i}$. Consequently,

$$
W=\left(M_{0} \times(-\infty, 0)\right) \cup\left(\bigcup_{i=1}^{\infty} W_{i}\right)
$$

is an $(n+1)$-manifold equipped with a lamination. It follows routinely that $\pi_{1}(W)$ is the direct limit of the inclusion-induced sequence

$$
\left\{\pi_{1}\left(\left(M_{0} \times(-\infty, 0)\right) \cup\left(\bigcup_{i=1}^{m} W_{i}\right)\right) \rightarrow \pi_{1}\left(\left(M_{0} \times(-\infty, 0)\right) \cup\left(\bigcup_{i=1}^{m+1} W_{i}\right)\right)\right\},
$$

namely, $G / P$. Hence, $W$ cannot be homotopy equivalent to a finite complex.

\section{REFERENCES}

[1] M. Bestvina and N. Brady, Morse theory and finiteness properties of groups, preprint.

[2] R. J. Daverman and F. C. Tinsley, The homotopy type of laminated manifolds, Proc. Amer. Math. Soc. 96 (1986), 703-708. MR 87e:57024

[3] L Laminations, finitely generated perfect groups, and acyclic mappings, Michigan Math. J. 33 (1986), 343-351. MR 87k:57016

Department of Mathematics, University of Tennessee-Knoxville, Knoxville, TenNESSEE 37996-1300

E-mail address: daverman@novell.math.utk.edu

Department of Mathematics, The Colorado College, Colorado Springs, Colorado 80903

E-mail address: ftinsley@cc.colorado.edu 